\title{
Compatibilism, Indeterminism, and Chance
}

\section{PENELOPE MACKIE}

\begin{abstract}
Many contemporary compatibilists about free will and determinism are agnostic about whether determinism is true, yet do not doubt that we have free will. They are thus committed to the thesis that free will is compatible with both determinism and indeterminism. This paper explores the prospects for this version of compatibilism, including its response to the argument (traditionally employed against incompatibilist accounts of free will) that indeterminism would introduce an element of randomness or chance or luck that is inimical to free will and moral responsibility.
\end{abstract}

\section{Introduction}

Introductory discussions of the free will problem typically give a sketch of the terrain along the following lines. Compatibilists - that is, theorists who hold that free will is compatible with determinism - have the task of showing how free will could be compatible with determinism. Most obviously, they have the task of responding to versions of an Alternative Possibilities Argument, exemplified by the following:

Free will requires alternative possibilities. Determinism rules out alternative possibilities. So determinism rules out free will. ${ }^{1}$

\footnotetext{
${ }^{1}$ Versions of the Alternative Possibilities Argument represent only the most obvious type of objection to compatibilism. For other objections, see, for example, Michael McKenna and D. Justin Coates, 'Compatibilism', in E. Zalta, ed., The Stanford Encyclopedia of Philosophy, 2015, URL = $<$ https://plato.stanford.edu/archives/win2016/entries/compatibilism/>, §2; Kadri Vihvelin, 'Arguments for Incompatibilism', in E. Zalta, ed., The Stanford Encyclopedia of Philosophy, 2011, $\mathrm{URL}=\langle$ https://plato.stanford.edu/archives/fall2015/entries/incompatibilism-arguments/>.
} 
By contrast, according to this typical sketch, Libertarians (that is, incompatibilists who also hold that we have free will) have the task of demonstrating how the indeterminism that they think necessary for free will could actually be compatible with free will. In particular, they have the task of responding to versions of an Indeterminism and Control Argument, exemplified by the following:

Free will requires not only alternative possibilities, but also control over which of the alternative possibilities comes about. Indeterminism allows for alternative possibilities, but rules out control over which of them comes about. So indeterminism rules out free will.

The two problems may be encapsulated more frivolously in verse form, as follows: ${ }^{2}$

\section{Worries about Determinism}

There was a young man who said, 'Damn!

It appears to me now that I am

Just a being that moves

In predestinate grooves,

Not a taxi or bus, but a tram.'

Worries about Indeterminism (bowdlerized version)

There's another young man who said, 'Yuk!

I'm a vehicle running amok!

Now I've gone off the rails

I can see this entails

That my path is determined by luck.'

This typical sketch of the tasks facing the compatibilist and libertarian is, however, too simplistic, for the following reason. Most contemporary compatibilists are what I call 'two-

\footnotetext{
${ }^{2}$ The first of these is a version of limerick that, according to the Oxford Dictionary of Quotations, was originally composed by Maurice E. Hare. The second limerick is my own contribution to the genre.
} 
way compatibilists'. That is, in addition to holding that free will is compatible with Determinism (the defining feature of compatibilism) they also hold - for reasons that I shall explain - that free will is compatible with Indeterminism. ${ }^{3}$ These two-way compatibilists therefore confront not just one task, but two:

Task 1: Reconciling free will with Determinism.

Task 2: Reconciling free will with Indeterminism.

Before proceeding, I must explain my use of some relevant terminology.

By 'free will' I shall mean 'the ability (or power or capacity) to act freely, in a sense of acting freely that is relevant to moral responsibility'. ${ }^{4}$ This characterization is deliberately neutral as between compatibilist and incompatibilist accounts of free will and free action. By 'free agency' I shall mean 'the possession or exercise of this ability'.

Following other writers, I take Determinism to be:

'The thesis that there is at any instant exactly one physically possible future.' (van Inwagen ...) More fully, at any instant exactly one future is compatible with the state of the universe at that instant and the laws of nature. ${ }^{5}$

By 'Indeterminism' I simply mean the negation of Determinism. It follows from this that one or other of Determinism or Indeterminism must be true: there is no 'third way'. Note

\footnotetext{
${ }^{3}$ For the explanation of why I now capitalize the initial letters of the words 'Determinism' and 'Indeterminism', see the penultimate paragraph of this section.

${ }^{4}$ For similar characterizations, see Alfred Mele. Free Will and Luck (New York: Oxford University Press, 2006), 17; Vihvelin, ‘Arguments for Incompatibilism’, §1; also McKenna and Coates, 'Compatibilism', §1.

${ }^{5}$ Mele, Free Will and Luck, 3. The characterization quoted by Mele is from Peter van Inwagen, An Essay on Free Will (Oxford: Clarendon Press, 1983), 3.
} 
also that Indeterminism, so defined, does not imply that everything that happens is random or chancy (whatever that might mean). All that it implies is that there are some undetermined events: i.e., events that are not determined by the past plus the laws of nature. I use 'deterministic world' to mean 'world of which Determinism is true', and 'indeterministic world' to mean 'world of which Indeterminism is true'. (Thus we may ask, for example, whether our world is deterministic or indeterministic, whether there are possible worlds that are deterministic in which agents act freely, and so on.)

Finally, although I shall always use 'Indeterminism', with a capital 'I', to designate the 'global' doctrine that is the negation of Determinism, ${ }^{6} \mathrm{I}$ shall also use 'indeterminism' (with lower-case ' $i$ ') in a sense that allows that there may be different types (or kinds) of indeterminism in worlds of which Indeterminism is true: different ways in which a world might be an indeterministic world. (What I mean by 'different types of indeterminism' will become clear in $\S \S 2-3$.)

With this terminology established, 'two-way compatibilism' may be characterized as the view that agents can act freely both in deterministic worlds and also in indeterministic worlds, and hence incurs the two 'Tasks' that I identified above.

\section{Two-way compatibilism and Task 2}

Given that most contemporary compatibilists are two-way compatibilists, it may seem initially surprising that, in discussions of compatibilism in the contemporary free will literature, the focus is still almost exclusively on Task 1 . Very little attention is paid to how compatibilists should handle Task 2.

It is true that introductions to the free will problem often raise the question why so much attention is still paid to whether compatibilism is defensible, given that so many theorists,

\footnotetext{
6 'Global' in the sense that it applies to the world as a whole. For parity, I use 'Determinism' (with capital 'D') for the (global) thesis that I have defined in this section.
} 
including many compatibilists themselves, doubt or deny that Determinism is true. After all, if Determinism is actually false of our world, as many believe, why would it matter whether agents can have free will in deterministic worlds? Wouldn't the question be of merely academic or theoretical interest? Yet the issue of the defensibility of compatibilism is usually presented as if it were highly relevant to the question whether we - actual human agents - actually have free will.

The standard response to this question - about the contemporary relevance of compatibilism - is that even if Determinism is false of our world, something close enough to Determinism might be true to make the worries traditionally raised about the compatibility of free will with Determinism relevant to whether we have free will. ${ }^{7}$ However, although this may explain why contemporary discussions continue to treat the question whether compatibilists can fulfil Task 1 as significant, it does not appear to explain the neglect of Task 2 .

The most likely explanation for this neglect is an assumption (seldom stated) that the two-way compatibilists' version of Task 2 is a relatively trivial one. In particular, it may be assumed that, unlike the version of this task that the libertarian faces, it does not make the compatibilist vulnerable to what I called, in the opening section, 'the Indeterminism and Control Argument'. Why so? Well, it may be said, the two-way compatibilists' Task 2 is simply the task of showing that agents can act freely in indeterministic worlds. By contrast, the task that the libertarian faces is much more demanding. As McKenna and Pereboom put it:

\footnotetext{
${ }^{7}$ See, for example: Michael McKenna and Derk Pereboom, Free Will: A Contemporary Introduction (New York and London: Routledge, 2016), 23-24; Robert Kane, A Contemporary Introduction to Free Will (Oxford \& New York: Oxford University Press, 2005), 8-10; Gary Watson, ed., Free Will (Oxford: Oxford University Press, $2^{\text {nd }}$ edition, 2003), Editor's Introduction, 9-10; Ted Honderich, How Free Are You? The Determinism Problem (Oxford \& New York: Oxford University Press, 1993), 66, 79.
} 
$[F]$ or those who believe determinism undermines free will, it is not enough [to allow for free will] that indeterminism is true. It must be that the way indeterminism is true leaves indeterministic breaks in the relation between events in just the right places - in particular, just where free actions occur. ${ }^{8}$

On the face of it, two-way compatibilists incur no such commitment concerning the locus of the 'indeterministic breaks' or undetermined events in indeterministic worlds in which there are free agents. This difference is obscured in simple statements of the Indeterminism and Control Argument such as the one I provided in the opening section. On reflection, one can see that what drives the argument (and similar arguments against libertarianism) is not the idea that indeterminism somewhere in the world would rule out control, but rather that indeterminism concerning the alternative possibilities that are regarded by the libertarian as crucial to free will would rule out control. ${ }^{9}$

In what follows, however, I shall argue that matters are not so simple. In particular, I shall maintain that two-way compatibilists cannot so easily evade versions of the Indeterminism and Control Argument that they are so fond of pressing against the libertarian. I shall argue that they need to take seriously what I shall call 'the challenge' (to two-way compatibilism):

The challenge:

Can two-way compatibilists fulfil Task 2 (reconciling free will with Indeterminism) without undermining their case against libertarians?

\footnotetext{
${ }^{8}$ McKenna and Pereboom, Free Will, 17 (italics in the original).

${ }^{9}$ Cf. Alfred Mele, 'Libertarianism, Compatibilism, and Luck', Journal of Ethics 19 (2015), 1-21; $14-15$.
} 
Although I believe that this challenge can be met, I also think that it is by no means a trivial one. That is the main thesis that I hope to establish in this paper.

\section{Two-way compatibilism and 'irrelevant indeterminism'}

I have said that most contemporary compatibilists are two-way compatibilists. The crucial point is that most contemporary compatibilists do not commit themselves to the truth of Determinism, and yet believe that we have free will. Most contemporary compatibilists (i) are either agnostic about whether Determinism is true, or believe that Determinism is false (and hence that Indeterminism is true); and also (ii) are what I shall call 'optimistic compatibilists': that is, they believe that we do have free will. Evidently, then, they must, if they are to be consistent, be two-way compatibilists.

In addition, I think that all compatibilists should be two-way compatibilists. They should be so even if, unlike most contemporary compatibilists, they are firmly of the opinion that Determinism is true (and hence are committed 'soft determinists', according to the standard terminology). The reason is that the alternative version of compatibilism, 'one-way compatibilism', seems grossly implausible.

'One-way compatibilism' is the view that free will is compatible with Determinism, but incompatible with Indeterminism. Hence one-way compatibilism entails that agents cannot act freely in indeterministic worlds. But this seems absurd. There are many features that could make a world an indeterministic one, and yet which, it seems, no one could sensibly suppose would undermine the freedom of agents in that world. Consider undetermined events that fall into one of the following categories:

Type 1: Undetermined events that are spatially and causally isolated from the agents and their actions. 
Type 2: Undetermined events that occur long before the agent's birth, even if part of a causal chain leading to the agent's existence (and hence to the agent's action).

Type 3: Undetermined events whose occurrence is a necessary condition for the agent's staying alive long enough to perform any action at all.

Many, if not all, of such events appear to be irrelevant to free will, as the following examples show.

Type 1: Suppose that there are some undetermined events, but they occur only in distant galaxies, or at the bottom of the ocean. This would mean that Determinism is false - and that the world is an indeterministic world. Yet, on the assumption that these events are causally isolated from human actions, surely no one could think that this kind of indeterminism could be relevant to whether human beings act freely.

Type 2: Suppose that it was genuinely a matter of chance that my parents ever met - not in the everyday sense of 'chance', but that their meeting was genuinely an undetermined event. Could anyone think that this fact - this indeterminism in the way in which I came into existence - could be relevant to whether I have ever acted freely? Surely not.

Type 3: Suppose that my friend Alan decided, last Tuesday morning, at 10 a.m., to buy a book. And suppose that, although he made that decision, there was a genuine chance that he wouldn't do so, for the following reason: as he was deliberating about what to buy, it was genuinely consistent with the past up until that time, plus the laws of nature, that he might drop dead before the end of his deliberation, and hence not make any decision at all. In other words, suppose that Alan's staying alive long enough to make the decision was 
something that was undetermined. Could anyone seriously think that that type of indeterminism (in the process that led to Alan's decision) would mean that the decision that he actually made was not a free one? Surely not. Should a compatibilist say, to Alan, after the event: 'You didn't act freely when you decided to buy that book, because you might have dropped dead before you made up your mind'? That seems absurd. The compatibilist should admit that, if there really was such indeterminism in the process leading to Alan's decision, it was irrelevant to the question whether Alan acted freely in making it. Admittedly, such indeterminism would not exactly be freedom-enhancing. But it certainly doesn't seem to be freedom-prohibiting.

To sum up: all three of these examples of indeterminism seem to be ones that a compatibilist should regard as irrelevant to freedom - irrelevant because they are obviously not 'freedom-prohibiting'. That is, if one thinks that it is possible for agents to act freely at all (as compatibilists do), one should not think that the existence of these three types of indeterminism undermines their freedom of action. ${ }^{10}$ (It is worth noting that 'irrelevant indeterminism' cannot be equated with 'indeterminism that is isolated from the causal history of the agent's action'. As the second and third examples show, such isolation is not necessary for irrelevance, even if it is sufficient. ${ }^{11}$ )

\footnotetext{
${ }^{10}$ Of course, there are those who think that free will is impossible, regardless of whether Determinism is true. But these theorists ('impossibilists') should still not think that these types of indeterminism are freedom-prohibiting in a sense of taking away a freedom that would or might otherwise obtain. For the term 'impossibilism', and a discussion of some versions of this view, see Vihvelin, 'Arguments for Incompatibilism'.

${ }^{11}$ My argument in this section obviously raises the question why any compatibilists have ever been one-way compatibilists. Yet the literature is replete with references to classical compatibilists who are attributed this position (allegedly holding that free will is not only compatible with, but also requires, Determinism). I cannot pursue this intriguing question here. But one possibility is that at least some of these theorists intended to hold, not that free will requires that the world as a whole be deterministic, but the more restricted thesis that free actions must be deterministically caused.
} 


\section{Is there an easy answer to 'the challenge'?}

The remarks I've just made may seem to reinforce the suggestion that two-way compatibilists have a very easy way of responding to what I have called 'the challenge', without making any concessions to the libertarian.

Two-way compatibilists must say that some kinds of indeterminism are 'freedomconsistent'. (That is, after all, what defines them as two-way rather than one-way compatibilists.) But they can still say that some other kinds of indeterminism are 'freedomprohibiting'. Suppose, then, that there is a kind of indeterminism that libertarians regard as 'freedom-enabling' (i.e., as necessary for free will and free agency) but which the two-way compatibilist regards as 'freedom-prohibiting' (i.e., incompatible with free will and free agency). If correct, this hypothesis provides the two-way compatibilist with an easy answer to 'the challenge'. And the plausibility of the hypothesis may seem to be supported by the fact that the types of indeterminism mentioned in the previous section seem irrelevant to freedom not merely from the point of view of a sensible compatibilist, but also from the point of view of a libertarian.

In order to evaluate this hypothesis, however, we must first identify exactly what type of indeterminism libertarians do regard as necessary for free will - as 'freedom-enabling'. This is the task of the next section.

\section{L-indeterminism}

Let us consider what type of indeterminism the typical libertarian regards as a necessary condition for free will. I call this 'L-indeterminism'. The typical libertarian (henceforth simply 'the libertarian') holds the principle (L): 
(L) An action $A$ performed at $t$ is (directly) free only if it satisfies the following condition:

(U) There is another possible world with the same past and same laws of nature up to $t$ in which, at $t$, the agent does something other than $A .^{12}$

A few comments on this are in order. First, the qualification 'directly' reflects the fact that some libertarians hold that an action can be free (indirectly free) if it is the result (at least in an appropriate way) of a previous directly free action. Secondly, for simplicity, I shall treat decisions and choices as belonging in the class of actions. Thirdly, the 'does something other than $A^{\prime}$ in condition (U) is to be interpreted in such a way that an involuntary 'action' such as dropping dead is not to count as 'doing something'. That is, I take it, one of the lessons of my example of Alan and his book purchase. ${ }^{13}$

Obviously, principle (L) involves an incompatibilist conception of the 'could have done otherwise' that many regard as a requirement for free agency. According to (L), an action done at time $t$ is (directly) free only if the agent could have done otherwise, in a sense that entails that the agent's acting differently at $t$ is consistent with the past up to $t$ and the laws of nature.

Return to Alan and his decision, at 10 a.m. last Tuesday, to buy a book. The libertarian who holds principle (L) will say that if Alan's decision was a (directly) free one, then it

\footnotetext{
${ }^{12}$ Cf. Mele, Free Will and Luck, 58. Principle (L) is related to what Robert Kane has called 'the Indeterministic Condition' that typical libertarians insist on as a requirement for free action (Kane, Contemporary Introduction, 38).

${ }^{13}$ For the same reason, I have deliberately used 'does something other than $A$ ', rather than 'does not do $A$ ' in (U). It should be noted, though, that 'doing something other than $A$ ', where doing $A$ is making a decision to do $C$, does not entail 'making a decision to do not- $C$ '. It could, for example, include deliberately (or at least voluntarily) postponing making a decision about whether to do $C$. (For emphasis on this point, see Helen Steward, 'The Truth in Compatibilism and the Truth of Libertarianism', Philosophical Explorations 12 (2009), 167-179; also Helen Steward, A Metaphysics for Freedom (Oxford: Oxford University Press, 2012), 154-156. But see also note 28 below.)
} 
was genuinely undetermined, by the past and laws of nature, up until 10 a.m. on Tuesday, whether he would make that decision. And not because it was genuinely undetermined whether he would stay alive to do anything at all, but genuinely undetermined whether he would make that decision rather than (voluntarily) doing something else instead.

In more detail: the principle (L) may be seen as incorporating the following:

(L1) An agent directly acts freely in doing $A$ at $t$ (the agent's action $A$ at $t$ is directly free) only if both:

(a) the agent's doing $A$ at $t$ is itself an undetermined event (in the sense of an event that is undetermined by the past up to just before $t$, plus the laws of nature); and

(b) there is some other action $B$, such that it was undetermined by the past up to just before $t$ plus the laws of nature whether the agent would do $A$ at $t$ or $B$ at $t{ }^{14}$

Some libertarians add:

(L2) An agent acts freely in doing $A$ at $t$ (the agent's action $A$ at $t$ is free) only if EITHER: the agent's doing $A$ at $t$ is a directly free action (one that satisfies the conditions (a) and (b) of (L1));

OR: the agent's doing $A$ at $t$ is appropriately related to some earlier directly free action of the agent.

Once the libertarian's commitment to (L) is made explicit, it might be supposed (in line with the hypothesis suggested in the previous section) that the disagreement between the two-way compatibilist and the libertarian concerning the relation of free will to Indeterminism consists in the following. The libertarian thinks that the satisfaction of principle (L) is a requirement for (directly) free action. The indeterminism that $(\mathrm{L})$ requires is not simply that the world be indeterministic, but also that the agent's action be itself an undetermined event that satisfies (a) and (b) of (L1). By contrast (according to this

\footnotetext{
${ }^{14}$ As before, 'action' must be interpreted so as to exclude involuntary 'actions' such as dropping dead.
} 
hypothesis), the two-way compatibilist will hold that, in order to be a free action, an action must not be one of which both (a) and (b) are true. (It might be an action that satisfies condition (a) of (L1), ${ }^{15}$ but it must not satisfy condition (b) as well.)

In $§ 7$ I shall question whether this is, in fact, the appropriate stance for the two-way compatibilist to take. But to have a clearer sense of what is at issue, let us next consider what problems the libertarian's commitment to (L) appears to confront.

\section{Problems for the compatibility of free will and L-indeterminism}

If L-indeterminism (characterized as 'the indeterminism needed for the satisfaction of principle (L)') is what libertarians think free will requires, their view faces well-known problems: problems that suggest that this kind of indeterminism would actually undermine free will, by introducing an element of randomness or chance or luck that is incompatible with free and responsible agency. ${ }^{16}$

There are various versions that this problem can take. I'll focus on two, which I call 'the problem of control' and 'the problem of luck'.

The problem of control says that if an action satisfies condition (U), then the agent is not in control of the fact that the action occurs. Combined with the plausible assumption that unless an agent is in control of whether an action occurs, the action is not free, the problem of control leads to the conclusion that, far from providing a way in which actions can be free, the indeterminism required for condition (U) would actually prevent an action from being free.

The problem of luck says that if an action satisfies condition (U), then it is a matter of luck that the agent performs that action rather than some alternative action. Combined with

\footnotetext{
${ }^{15}$ Bear in mind that Alan's decision, in my example in $\S 3$, might be an action that satisfies (a).

${ }^{16}$ Recall the association between free will and responsibility in my initial characterization of free will (in §1).
} 
the plausible assumption that if this a matter of luck, the action is not free, the problem of luck again leads to the conclusion that the indeterminism required for condition (U) would prevent an action from being free. ${ }^{17}$

\subsection{L-indeterminism and the problem of control}

I'll illustrate what I call 'the problem of control' using an example taken from Peter van Inwagen's introductory book Metaphysics. ${ }^{18}$ Van Inwagen says:

Let us look carefully at the consequences of supposing that human behavior is undetermined. Suppose that Jane is in an agony of indecision; if her deliberations go one way, she will in a moment speak the words, "John, I lied to you about Alice," and if her deliberations go the other way, she will bite her lip and remain silent. We [are supposing] that there is a physically possible future in which each of these things happens ... Let us suppose that there is a certain current-pulse that is proceeding along one of the neural pathways in Jane's brain and that it is about to come to a fork. And let us suppose that if it goes to the left, she will make her confession, and that if it goes to the right, she will remain silent. And let us suppose that it is undetermined which way the pulse will go when it comes to the fork ...

Now let us ask: Does Jane have any choice about whether the pulse goes to the left or the right? ... it is very hard to see how she could have any choice about that ... There is no way for Jane to influence the pulse. There is no way for her to make it go one way rather than the other . . . and leave the "choice" it makes an undetermined event. If Jane did something to make the pulse go to the left, then, obviously, its going to the left would not be an undetermined event. ${ }^{19}$

\footnotetext{
${ }^{17}$ The term 'problem of luck' is sometimes used in the literature to include what I call 'the problem of control' as well as what I call 'the problem of luck'. For reasons that should become apparent, I prefer to distinguish the problems.

${ }^{18}$ Peter van Inwagen, Metaphysics (Oxford: Oxford University Press, 1993).

${ }^{19}$ Van Inwagen, Metaphysics, Ch. 11, 192-193 (italics in the original).
} 
I have depicted the situation in the diagram below. Suppose that Jane makes the decision to speak (DS), and does so freely, according to the libertarian. According to the libertarian view we are considering, this implies that, up until the moment of decision, it is undetermined, by the past and laws of nature, whether she will decide to speak, or do something else. We also suppose (for the sake of the example) that the 'something else' is deciding to remain silent (DQ).

Author's note to Editor: please see separate document for a more detailed version of Figure 1.

\section{Jane decides to speak} (DS)

State of world

before $t$

(plus laws of nature)

\section{Jane speaks}

(S)

Jane remains silent (Q)

$$
\begin{aligned}
& \text { Jane decides to remain silent } \\
& \text { (DQ) } \\
& \uparrow \\
& \text { time } t \text { (the time for decision) }
\end{aligned}
$$

\section{Figure 1}

According to the libertarian view we are considering:

(i) Both DS and DQ are physically possible continuations of the state of the world before $t$.

(ii) When Jane decides (at $t$ ) to speak, Jane's decision (DS) is a free action.

Since Jane's decision DS is a free action:

(iii) When Jane decides (at $t$ ) to speak, Jane controls which of DS and DQ comes about. 
But this combination of views confronts an objection. The objection is that (i) and (iii) are inconsistent. How could Jane control which of DS and DQ comes about, given the assumption that both DS and DQ are physically possible continuations of the state of the world before $t$ ? Here, of course, it is important to note that the state of the world before $t-$ the state that, ex hypothesi, does not determine which of DS and DQ comes about includes all the facts about Jane, including her mental states - which in turn includes her preferences, desires, intentions, etc., up to $t$. Hence, if Jane does somehow control the outcome in this example, she must do so in a way that leaves those prior-to- $t$ mental states intact.

The objection may be put in terms of the following simple argument, adapted from van Inwagen's discussion:

(1) The outcome of an indeterministic process is not determined by anything.

(2) To control the outcome of an indeterministic process would be to determine the outcome.

Therefore:

(3) It is not possible to control the outcome of an indeterministic process. ${ }^{20}$

So, in particular, Jane cannot control the outcome of the indeterministic process that (in our example) actually leads to DS - although DS is, according to the libertarian, supposed to be a free decision of Jane's.

So far, the libertarian's position may seem hopeless. However, as van Inwagen is well aware, some libertarians respond to this argument by appeal to a special kind of causation - agent-causation. ${ }^{21}$ Such agent-causal libertarians may argue as follows:

\footnotetext{
${ }^{20}$ Cf. van Inwagen, Metaphysics, Ch. 11, 193. Van Inwagen does not, however, use the word 'control' in his presentation of the argument, but speaks instead of whether the agent 'has a choice about' whether the outcome occurs.

${ }^{21}$ Cf. van Inwagen, Metaphysics, Ch. 11, 193-194.
} 
'There are two senses of 'indeterministic process'. In one sense (Sense 1) an indeterministic process is one whose outcome is not determined by anything. In another sense (Sense 2), an indeterministic process is one whose outcome is not determined by preceding events and states of the world plus laws of nature. We may grant premise (2) of the argument above - that to control the outcome would be to determine the outcome. We thus agree that an agent cannot control the outcome of an indeterministic process in Sense 1. Nevertheless, an agent can control the outcome of an indeterministic process in Sense 2. Why so? Because an agent is not an event or state. And an agent can determine an outcome (by causing the outcome to occur, in an act of agent-causation) even if the outcome is not determined by previous events or states (including previous events or states involving the agent).'

This appeal to agent causation - as a way in which agents may determine outcomes that are otherwise undetermined - is notoriously controversial. ${ }^{22}$ Even many libertarians regard it as unsatisfactory. ${ }^{23}$ Although I cannot pursue the issue here, it is fair to say that the 'problem of control' illustrated here is generally recognized to be a serious challenge to libertarians. And the problem arises, in part, because of the libertarian's insistence on condition (U) in principle (L).

\subsection{L-indeterminism and the problem of luck}

As well as facing this problem of control, L-indeterminism also confronts a problem of luck. The problem can be illustrated by considering a variant of the previous example, in which, instead of two possible decisions (DS and DQ) for Jane in the actual world, we

\footnotetext{
${ }^{22}$ For discussion of agent causal libertarianism, see Gary Watson, ed., Free Will ( $2^{\text {nd }}$ edition, 2003). ${ }^{23}$ Including van Inwagen (Metaphysics, 194). Contemporary philosophers who have attempted to defend libertarianism without appeal to agent causation include Robert Kane. See, for example, his 'Responsibility, Luck, and Chance: Reflections on Free Will and Indeterminism', Journal of Philosophy 96 (1999), 217-240.
} 
consider the contrast between the actual world, in which Jane makes the decision DS, and a possible world that is a duplicate of the actual world up until just before time $t$, and has the same laws of nature as the actual world, and yet in which Jane (or a 'Twin-Jane' who is a duplicate or counterpart of Jane; it makes no difference) makes the opposite decision DQ. The libertarian must say that, if the availability of the alternative decision (DQ) in the actual world was required for Jane's actual decision (DS) to be (directly) free, then a world in which Twin-Jane makes that alternative decision is a possible one. We thus have the setup represented in the diagram below:

Author's note to Editor: please see separate document for a more detailed version of Figure 2.

\section{ACTUAL WORLD JANE}

Jane decides to speak

(DS)

State of world

before $t$

(plus laws of nature)
Jane speaks

(S)

POSSIBLE WORLD TWIN-JANE

State of world

before $t$

(plus laws of nature)
Twin-Jane decides to remain silent (DQ)

$\uparrow$

time $t$ (the time for decision)
Twin-Jane

remains silent

(Q)

Figure 2 
The existence of the possible world (which the libertarian seems bound to admit) appears to be an embarrassment to the libertarian. Given the possibility of the world in which Twin-Jane makes the decision DQ, how can it be other than a matter of luck that DS comes about in the actual world rather than DQ? Adapting the words of Mele's discussion of a similar case:

If there is nothing about [the agents'] powers, capacities, states of mind, moral character, or the like in either world that accounts for this difference, then the difference seems to be just a matter of luck. And given that neither world diverges from the other in any respect before $t$, there is no difference at all [between Jane and Twin-Jane] in these two worlds to account for the difference in [their] decisions. ${ }^{24}$

This problem of luck (if indeed it is a problem) seems to arise even for the libertarian who adopts the agent-causal response to the previous 'problem of control'. Suppose we grant, for the sake of argument, that in the actual world Jane does determine that DS occurs, by agent-causing it. (She is the 'undetermined determiner' of DS, according to the agentcausal theorist.) For the sake of parity, we should say that Twin-Jane, in the alternative possible world, determines that DQ occurs, again by agent-causing it. But then why isn't it a matter of luck that Jane agent-causes (and determines) DS rather than DQ? ${ }^{25}$

\section{Two-way compatibilism and L-indeterminism}

We now have a characterization of a type of indeterminism - L-indeterminism - that the libertarian regards as necessary for free agency (because it is necessary for the satisfaction

\footnotetext{
${ }^{24}$ Mele, Free Will and Luck, 9.

${ }^{25}$ See Alfred Mele, 'Libertarianism, Luck, and Control', Pacific Philosophical Quarterly 86 (2005), 395-421, and Randolph Clarke, 'Agent Causation and the Problem of Luck', Pacific
} Philosophical Quarterly 86 (2005), 408-421. 
of the libertarian principle (L)), and yet which (in the light of the problems of luck and control) may appear also to be incompatible with free agency.

Now, of course, the compatibilist cannot accept the principle (L), since (L) entails that free action is incompatible with Determinism. In other words, compatibilists cannot accept that L-indeterminism is what I have called 'freedom-enabling' - that it must be involved in anything that counts as a (directly) free action.

But it does not follow, from this, that the two-way compatibilist must say that Lindeterminism is never involved in free action. There are, at least in theory, two possible options for the two-way compatibilist, Option A and Option B.

Option A: L-indeterminism is freedom-prohibiting (although some other kinds of indeterminism are not). The libertarian is wrong both in thinking that L-indeterminism is freedom-consistent, and also in thinking that L-indeterminism is freedom-enabling (necessary for free will and free agency).

Option B: L-indeterminism is freedom-consistent. But the libertarian is wrong in thinking that L-indeterminism is not only freedom-consistent, but also freedom-enabling (necessary for free will and free agency).

We might describe compatibilists who take Option A as 'recalcitrant two-way compatibilists', and those who take Option B as 'liberal two-way compatibilists'.

It might seem obvious that the two-way compatibilist should take Option A, thus entirely avoiding the problems of control and luck presented in the previous section. This corresponds to the suggestion mentioned earlier in this paper (§4) concerning the two-way compatibilist's response to what I called 'the challenge' of reconciling free will with Indeterminism. 
In spite of this, though, I don't think it is obvious that the compatibilist should go for Option A rather than Option B. There are two reasons for this.

\section{First reason}

The first reason is that there appear to be plausible candidates for compatibilist sufficient conditions for free action that do not preclude that action's also satisfying condition (U) of the libertarian’s principle (L). Return to Jane in our example in $\S 6$ above. We can envisage that Jane takes herself to have good reasons to speak, and also good reasons to remain silent. After all, if she didn't think that was her situation, why would she be 'in an agony of indecision'? But if, whichever of these things she does, it will be a decision for which she has (or takes herself to have) good reasons, why shouldn't the two-way compatibilist say that, whichever way she decides, she does so freely - at least if certain other standard compatibilist conditions are fulfilled - that Jane is not coerced, not manipulated, not under

the influence of psychological compulsion, and so on? In other words, why shouldn't the (two-way) compatibilist regard Jane as an agent who satisfies (compatibilist) sufficient conditions for acting freely, regardless of the L-indeterminism involved?

\section{Second reason}

There is a further consideration that suggests that two-way compatibilists not only may, but also should, adopt Option B, at least if they are also what I have called 'optimistic compatibilists' (§3). Optimistic compatibilists, we may recall, do not doubt that we have free will, although they may be agnostic about whether our world is one of which Determinism is true, or one of which Indeterminism is true.

But now, what right does an optimistic compatibilist who either doubts or denies that Determinism is true have to be sure that, among the typical candidates for free actions that we perform, there aren't some that are undetermined events that satisfy condition (U)? 
Perhaps, for all we know, some of them do fall into this category. Perhaps all of them do. But if it is epistemically possible that all the actions we perform that are plausible candidates for free actions satisfy condition (U), then retaining optimistic compatibilism is incoherent, unless it is conceded that an action can satisfy condition (U) and still be a free action.

Moreover, even if we suppose that it is epistemically possible only that some (rather than all) of the plausible candidates for our free actions fall under this heading, optimistic compatibilism, although coherent, would be somewhat precarious, if the optimistic compatibilist adopts Option A rather than Option B. Such a compatibilist would have to hold that although we can be sure (although one wonders how) that at least some of our actions are free, we might be badly mistaken about which of our actions fall into this category. For (according to Option A), whether or not they are free would depend, inter alia, on whether they don't or do satisfy principle (U). And it is hard to see how we could know which of them do not.

This is not to say that we have good reasons for supposing that the actions that we routinely take to be free actions are undetermined events that satisfy principle (U). The libertarian's insistence that the satisfaction of principle (U) is a requirement for free action makes their view precarious in this respect. But it is one thing to point out that we have no reason to think that this libertarian requirement is fulfilled by the actions we standardly regard as free, and another to claim that we have positive reasons for thinking that it is not.

It is worth emphasizing that this worry for the optimistic two-way compatibilist - the epistemic possibility of widespread L-indeterminism in the genesis of the actions that we typically regard as free - does not appear to be dispelled by pointing out that we can be confident that some kinds of indeterministic influence on our actions and decisions are not a feature of the world we inhabit, since they would result in a kind of randomness at the 
macroscopic level that we know, on empirical grounds, does not obtain. ${ }^{26}$ For it is not at all clear that the widespread presence of L-indeterminism would produce such randomness. To see this, it is important to note that principle (L), as stated, does not require that a free action be such that the alternatives undetermined by the past and laws of nature include options that it would be wildly eccentric for the agent to take, and would make it (from a non-libertarian point of view) a fantastic accident that the agent's actual choice is one that appears reasonable rather than bizarre or capricious. Hence, for example, compatibilists cannot legitimately suppose, as A. J. Ayer appears to do in 'Freedom and Necessity', that if L-indeterminism were true of a person's actions, those actions would be so unpredictable as to lead us to treat the person as a lunatic rather than a moral agent. ${ }^{27}$

It is even enough, for the satisfaction of principle (L), as I have stated it, that the 'something else' that the agent could have done at $t$, consistently with the past and the laws of nature up to $t$, be a matter of simply postponing the performance of an action of the type the agent actually performed at $t$, or perhaps even performing, at $t$, an action of the same type as the one actually performed, but in a different manner. And whatever the evidence that human behaviour is not typically chaotic or random, it does not appear to rule out the presence of L-indeterminism that has more modest effects such as these. ${ }^{28}$

To sum up: I agree with Helen Beebee's observation that:

\footnotetext{
${ }^{26}$ Concerning the absence of these kinds of randomness at the macroscopic level, see John Martin Fischer and Mark Ravizza, S. J., Responsibility and Control: A Theory of Moral Responsibility (Cambridge: Cambridge University Press, 1998), 253-254. See also Honderich, How Free Are You?, 66.

${ }^{27}$ A. J. Ayer, 'Freedom and Necessity', in his Philosophical Essays (London: Macmillan, 1954).

${ }^{28}$ If principle (L) can be satisfied merely by its being undetermined which token action of a given type the agent will do, this raises the question whether $(\mathrm{L})$ is, after all, strong enough to capture the openness of alternative possible choices that the typical libertarian has in mind as a requirement of free action. Unfortunately, I cannot pursue this issue here. (See also note 13 above.)
} 
... we cannot ... be confident that ... if indeterminism is true, it is somehow confined to the quantum realm in a way that doesn't render any relevant macroscopic or psychological processes indeterministic, too. . . it might, for all we know, turn out that quite a lot of what we do $i s$ undetermined by the past plus the laws. ${ }^{29}$

To this Beebee adds:

So, if we think that this would undermine free will, then we'll have to admit that we are not after all entitled to be confident that we routinely act freely. ${ }^{30}$

Applying modus tollens to Beebee's conclusion, the optimistic two-way compatibilist should conclude that L-indeterminism may actually be a feature of many of our actions that is freedom-consistent rather than freedom-prohibiting.

\section{Two-way compatibilism and the problems of control and luck}

Suppose, then, that two-way compatibilists do go for Option B. Then, of course, they must provide answers to the problems of control and luck. How are they to do that, without undermining their case against the libertarian?

It seems clear that, in response to the problem of control, the compatibilist (from now on this means 'the Option B two-way compatibilist', unless otherwise indicated) should jettison the notion that, in cases of free action where L-indeterminism is present, the agent manages somehow to determine the otherwise undetermined outcome. The question is why this won't play into the hands of the libertarian.

The compatibilist, we are now envisaging, says that an action can be free even if it satisfies condition $(\mathrm{U})$, and it was thus undetermined, up to the time of action, whether the

\footnotetext{
${ }^{29}$ Helen Beebee, Free Will: An Introduction (Palgrave Macmillan, 2013), 129 (italics in the original).

${ }^{30}$ Ibid. (italics in the original).
} 
agent would do that action or something else. The suggestion is that an action can satisfy this condition, and yet be free, even if it is not, strictly speaking, true that the agent controls or determines whether that action occurs or, instead, some other action. In other words, the two-way compatibilist is envisaging that, where it is the case that there are alternative possibilities of the kind involved in L-indeterminism, and the action is nevertheless free, its being a free action does not require control over which of the alternative possibilities comes about. According to this view, the typical libertarian is doubly mistaken: mistaken in holding that such control is a requirement of free action, and mistaken in thinking that L-indeterminism provides scope for such control.

The libertarian's appeal to undetermined alternative possibilities is typically motivated by the idea that unless there are such alternative possibilities, the agent cannot really determine what happens in a way that is required for free agency. By contrast, the two-way compatibilists' view can be that the alternative possibilities provided by condition (U) are not freedom-enabling, and need not even be freedom-enhancing. Two-way compatibilists can regard the indeterminism in question as, so far as it goes, a hindrance to freedom. In other words, according to the two-way compatibilist who takes Option B, we sometimes act freely not because of, but in spite of, L-indeterminism, just as we sometimes act freely in spite of the fact that we might have dropped dead before the time of action.

This last consideration bears on the contrast between the two-way compatibilist's attitude to L-indeterminism and that of Robert Kane's event-causal version of libertarianism. ${ }^{31}$ Kane rejects the agent-causal response to the problem of control in favour of a view according to which, whichever way perplexed Jane decides, she is responsible for the decision, although she may have 'diminished control over each option considered

\footnotetext{
${ }^{31}$ See, e.g., Kane, 'Responsibility, Luck, and Chance'.
} 
separately'. ${ }^{32} \mathrm{He}$ also concedes that 'indeterminism . . . does seem to diminish rather than enhance agents' voluntary control'. ${ }^{33}$ Nevertheless, Kane's view differs significantly from that of the Option B two-way compatibilist. For Kane, unlike the compatibilist, thinks that the presence of L-indeterminism is required for an action to be directly free. He therefore faces, as the compatibilist does not, the problem that his view makes free agency conditional on there being actions (and a sufficiency of such actions) that involve Lindeterminism. ${ }^{34}$

The two-way compatibilist who adopts Option B can, I have argued, handle the problem of control without capitulating to the libertarian. But what of the problem of luck? On the face of it, the Option B two-way compatibilist appears to confront a stark dilemma. Either L-indeterminism brings with it a degree of luck or chance that prevents the action from being free, or it does not. If it does, then Option B is incoherent. If it does not, then there is no challenge for the libertarian to face.

In reply, however, we can observe that there is an important difference in the resources available to the Option B compatibilist and the libertarian in responding to the problem of luck. The compatibilist may say that what makes it the case that the element of luck (if it is present) does not undermine freedom is that the action satisfies sufficient conditions for free action of a type that would be unacceptable to a libertarian - unacceptable because they can also be satisfied by actions in which no element of L-indeterminism is involved. Evidently this response is unavailable to the libertarian. Hence (whether or not the response is ultimately an adequate one) Option B compatibilists may consistently make this

\footnotetext{
${ }^{32}$ Kane, 'Responsibility, Luck, and Chance', 237 (italics in the original). Here I am assuming that the case of Jane exemplifies the class of 'Self-Forming Actions' that Kane regards as directly free actions, and whose occurrence is (according to his theory) necessary for any other actions to be free.

${ }^{33}$ Ibid. (italics in the original).

${ }^{34}$ For a forceful expression of scepticism about whether this condition is fulfilled, see Beebee, Free Will, 126-127.
} 
response without undermining their case against the libertarian's claim that Lindeterminism is not only consistent with, but actually required for, free agency.

\section{Conclusion}

The fact that two-way compatibilists who adopt Option B confront the problems of control and luck (and similar problems) need not, I have suggested, undermine their case against libertarianism. It does, however, mean that their appeal to such problems in arguing against the libertarian must be appropriately circumspect, and (for consistency), must not involve the claim - typical of traditional compatibilist attacks on libertarianism - that Lindeterminism is actually incompatible with free agency. It will not do, for example, for them to maintain the traditional compatibilist view exemplified in the 'Indeterminism and Control Argument' stated in the opening section of this paper.

On the other hand, if two-way compatibilists adopt Option A, they may, with consistency, continue to employ these traditional arguments against the libertarian. However, if I am right, this may make precarious the optimism that so many contemporary compatibilists display concerning our possession of free will in a world that may, for all we know, be indeterministic.

In any case, though, I hope to have shown that the problems of reconciling indeterminism with free will that have traditionally been regarded as unique to the libertarian position are much more pertinent to the defensibility of compatibilism than has typically been recognized. ${ }^{35}$

\footnotetext{
${ }^{35}$ It has recently been argued that compatibilism faces a 'problem of luck' analogous to the traditional problem for libertarians, but which arises even if supposedly free actions are causally determined. (See Neil Levy, Hard Luck: How Luck Undermines Free Will and Moral Responsibility (Oxford: Oxford University Press, 2011), Ch. 4; Mirja Pérez de Calleja, 'Crossworld Luck at the Time of Decision is a Problem for Compatibilists as Well', Philosophical Explorations 17 (2014), 112-125, and, for discussion, Alfred Mele, 'Libertarianism,
} 
University of Nottingham

penelope.mackie@nottingham.ac.uk

Compatibilism, and Luck', Journal of Ethics 19 (2015), 1-21.) It should be noted that this problem is distinct from the problems for compatibilists concerning luck and control that I have discussed here, which concern the implications for (two-way) compatibilism of the concession that free actions may not be causally determined. 